\title{
Prevalence of HIV and other infections and correlates of needle and syringe sharing among people who inject drugs in Podgorica, Montenegro: a respondent-driven sampling survey
}

Dragan Lausevic ${ }^{1}$, Senad Begic ${ }^{1}$, Boban Mugosa ${ }^{1}$, Natasa Terzic ${ }^{1}$, Zoran Vratnica ${ }^{1}$, Itana Labovic ${ }^{2}$ and Ivana Bozicevic ${ }^{3^{*}}$

\begin{abstract}
Background: People who inject drugs (PWID) have significantly higher rates of blood borne and sexually transmitted infections due to unsafe injecting practices and risky sexual behaviors.

Methods: We carried out an HIV bio-behavioral survey using respondent-driven sampling (RDS) in people who use drugs (PWID) in Podgorica, Montenegro in 2013 in order to determine the prevalence of HIV, hepatitis C (HCV), hepatitis B surface antigen ( $\mathrm{HBsAg}$ ) and risk behaviors. Data were analyzed using RDS Analyst and SPSS 12.0 to obtain prevalence estimates of key bio-behavioral indicators and assess correlates of needle and syringe sharing using multivariate logistic regression.
\end{abstract}

Results: A total of 402 PWID were recruited. HIV prevalence was $1.1 \%$, while the prevalence of HCV and HBsAg was $53.0 \%$ and $1.4 \%$, respectively. In the multivariate analysis, significant correlates of needle and syringe sharing in the past month were being older than 26 years, female, injecting drugs more than once per day, injecting in parks or on streets, not being able to obtaining free-of-charge sterile needles and syringes and reporting more than four partners in the past 12 months.

Conclusions: The results indicate that the HIV epidemic in PWID in Montenegro might still be at a low level, though the HCV epidemic is well-established.

Keywords: People who inject drugs, HIV, Hepatitis C, Survey, Montenegro

\section{Background}

People who inject drugs (PWID) have significantly higher rates of blood borne and sexually transmitted infections (STIs) due to unsafe injecting practices and risky sexual behaviors [1]. Unsafe sexual behaviors facilitate transmission of STIs to their sexual partners, thus increasing the risk of the spread of HIV and other STIs [2].

Montenegro, a former Yugoslav republic, is a small, newly independent country in southeastern Europe with a population of 620,000 people and a territory of around $13,000 \mathrm{~km}^{2}$. Podgorica, the capital of Montenegro, is the largest city with around 200,000 inhabitants, constituting

\footnotetext{
* Correspondence: Ivana.Bozicevic@lshtm.ac.uk

${ }^{3}$ World Health Organization Collaborating Centre for HIV Surveillance, School of Medicine, University of Zagreb, Zagreb, Croatia

Full list of author information is available at the end of the article
}

almost a third of a total population of Montenegro [3]. Podgorica is situated almost in the geographical center of the country.

Since the beginning of HIV case reporting in 1989 until the end of 2013, 153 HIV cases were reported of whom five (2\%) were reported as being due to injecting drug use, $42 \%$ as homosexual transmission and $44 \%$ as heterosexual transmission [4]. In the period 2005-2013, the number of newly reported cases of HIV ranged from 1.1 to 2.2 per 100,000 population.

HIV prevention interventions and harm reduction programs in PWID have been available in Montenegro since 2004 [5]. Currently, there are two drop-in centers for PWID in Podgorica established in 2010 and one center for Voluntary and Confidential HIV Counseling and Testing (VCCT) established in 2003. The services of the Center for 
Methadone Substitution Therapy have been available since 2005 while those of the Center for Rehabilitation of Drug Addicts since 2008. Drop-in centers are managed by two non-governmental organizations (NGOs) while the VCCT is operated by the Institute for Public Health (IPH). PWID can obtain sterile injecting equipment free-of-charge at drop-in centers and via outreach services, as well as at the premises of the Primary Health Care Center in Podgorica. The Global Fund to Fight AIDS, Tuberculosis and Malaria has been a major funding source for HIV prevention among key populations in Montenegro since 2006.

Integrated bio-behavioral surveys (IBBS) using respondentdriven sampling (RDS) were implemented in Podgorica in 2008 and 2011 and found an HIV prevalence of $0.4 \%$ in 2008 and $0.3 \%$ in 2011, while the prevalence of hepatitis C (HCV) was $53.6 \%$ and $55.0 \%$, respectively $[6,7]$.

The aim of this paper is to present the results of the IBBS carried out in PWID in Podgorica in 2013, specifically the prevalence of $\mathrm{HIV}, \mathrm{HCV}$, and hepatitis $B$ virus surface antigen (HBsAg), and behaviors relevant for transmission of $\mathrm{HIV}$ and $\mathrm{HCV}$, as well as correlates of sharing of needles and syringes for drug injection.

\section{Methods}

\section{Sampling}

We conducted a cross-sectional bio-behavioral survey among PWID in Podgorica using RDS [8,9]. Recruitment was initiated with five seeds (initial participants), of whom four were males and one female. Seeds were diverse in respect to age and a place of residence in the city. Seeds, as well as each participant who completed the survey, were provided with three coupons to be used in recruiting maximum of three eligible participants. Eligible participants were individuals older than 18 years living in Podgorica for at least 3 months during 12 months before the survey and who injected drugs for non-medical purposes in a month preceding the survey. Before agreeing to participate in the survey, participants were described the nature and characteristics of the survey and were asked for a verbal consent to participate. Following the consent, participants completed a questionnaire and were afterwards provided with pre-test HIV counseling and taken a sample of venous blood. Primary monetary incentive and three coupons for peer recruitment were given to participants after completion of the abovementioned steps of the survey. Participants were instructed to re-visit the survey site in 14 days in order to collect recruitment-related secondary incentives and test results. Post-test counseling was provided after issuing HIV, HCV, and HBsAg test results. Data collection lasted from November to December 2013.

A sample size calculation was designed to detect a $9 \%$ decline in needle and syringe sharing in the past month with $80 \%$ power and an alpha error of $5 \%$, from a value of $13.6 \%$ in the survey carried out in 2011. It was estimated that the sample size of 376 PWID was needed and that was rounded up to 400 participants.

\section{Behavioral questionnaire}

We used the same behavioral questionnaire as in the 2011 survey, with minor modifications. The questionnaire was based on a standardized behavioral questionnaire for PWID published by the Family Health International and was slightly modified and adapted to the country context [10]. It sought data on socio-demographic status, knowledge regarding the modes of HIV transmission, patterns of sexual and drug using behaviors, past HIV testing, and awareness and utilization of harm reduction services. A questionnaire was self-administered. In case that a participant needed help during completion of a questionnaire, trained study staff provided appropriate explanations and assistance. Data were also collected on participants' social network sizes that were assessed as the number of PWID they know by name, are older than 18 years, who lived in Podgorica, and have seen them in the past 3 months.

\section{Laboratory methods}

Blood samples were tested in the laboratory of the Center for Medical Microbiology of the IPH, which serves as a national referral center for HIV testing. Serum samples were tested for the presence of HIV-1/2 antibodies and p24 antigen using ELISA (HIV Ab\&Ag, Dia.Pro Diagnostic Bioprobes srl., Milano, Italy), while confirmatory HIV-1/2 testing was done by NEW LAV BLOT I and NEW LAV BLOT II (Bio-Rad, Marnes-la-Coquette, France). ELISA was also used for detection of antibodies against HCV (HCV Ab, Dia.Pro Diagnostic Bioprobes srl., Milano, Italy) and HBsAg (HBsAgone Version ULTRA, Dia.Pro Diagnostic Bioprobes srl., Milano, Italy).

All survey procedures were conducted by trained personnel. Ethical approval was granted by the Ethical Committee of the IPH in Podgorica.

\section{Analysis}

Univariate analysis to calculate key socio-demographic, behavioral, and biological indicators was done using Respondent-Driven Sampling Analyst (RDS-A) statistical software that provides weighted population estimates with $95 \%$ confidence intervals $(95 \% \mathrm{CI}$ ) of the variables of interests [11]. Bivariate and multivariate logistic regression analysis was done using SPSS 12 statistical software package on unweighted data (SPSS for Windows, Version 12.0). Data on seeds were excluded from the analysis, as per RDS analysis procedures.

We assessed factors associated with needle and syringe sharing in a month before participation in the survey using logistic regression analyses. Sharing needles and syringes was defined by either giving needles and syringes 
that a respondent used by himself/herself to other PWID or using needles and syringes for injecting drugs that were already used by other PWID. The following variables were considered potential correlates of needle and syringe sharing: age, gender, monthly income, frequency of injections in the past month, number of partners in the last 12 months, injecting drugs in an outdoors setting (in parks or on streets) during past month, and being given free-of-charge needles and syringes by NGOs and the Primary Health Care Centre in the past 12 months. Results are presented as odds ratios (ORs) with 95\% CI. Age and gender as confounders and variables associated with sharing needles and syringes at the level of $p<0.05$ in the bivariate analysis were included in the multivariate logistic regression model. The cutoff for considering a result to be statistically significant in the multivariate analysis was set up at $p<0.05$.

Missing values were excluded from the analyses.

\section{Results}

Recruitment patterns, socio-demographic characteristics, and knowledge of HIV

Five seeds recruited a total of 402 eligible participants. The median social network size was six (interquartile range $(\mathrm{IQR})=3-10)$. The mean number of waves was 8.2 , ranging from 4 to 10 . We distributed a total of 1,202 coupons.

A majority of survey participants were men (90.1\%). The median age of respondents was 32 (IQR 28-35) years. A majority of PWID completed either secondary (60.5\%) or primary $(23.5 \%)$ school. Being unemployed was reported by $58.2 \%$ of respondents, while $64.7 \%$ reported an income of $<150$ EUR per month.

A majority of respondents knew that it is possible to decrease the risk of HIV transmission by not sharing needles and other injecting equipment $(86.1 \%)$ and by proper and consistent use of condoms during sexual intercourse (85.4\%), but only $37.1 \%$ had comprehensive knowledge of HIV ${ }^{a}$.

\section{HIV testing and HIV, HCV, and HBsAg prevalence}

A majority of PWID (75.6\%) knew where it was possible to be tested for HIV.

Never been tested for HIV was reported by $57.7 \%$ of PWID while $18.9 \%$ were tested during the last 12 months.

HIV prevalence was $1.1 \%$, while the prevalence of $\mathrm{HCV}$ and HBsAg was $53.0 \%$ and $1.4 \%$, respectively. HCV prevalence was $31.5 \%$ in those younger than 25 years of age and $57.4 \%$ among older than 26 years.

\section{Sexual risk behaviors}

A majority of PWID (70.1\%) were sexually active during a month preceding the survey. A half of respondents reported having regular partners at the time of the survey, and $24.2 \%$ used condom at last intercourse with a regular partner (Table 1). Before having the first sexual intercourse with a current or last regular partner, only $28.6 \%$ of PWID discussed HIV status with that partner. Having non-regular partners during the past 12 months was reported by $62.2 \%$ of PWID, and $46.4 \%$ of these reported consistent condom use with this type of partners.

A substantial number (68.9\%) reported more than one sexual partner in the past 12 months while more than a third of PWID had four or more sexual partners in that time period.

Almost a half of PWID had experience of commercial sex, either in terms of paying or being paid for sex or exchanging sex for drugs, and $79.5 \%$ of these reported using a condom during the last commercial sexual intercourse.

A majority of participants (84.6\%) reported using drugs sometimes or often before sexual intercourse in the past 12 months.

\section{Drug use behaviors}

The median age of the first injecting drug use was 24 years $(\mathrm{IQR}=20-28)$, while the median duration of injecting drugs was 5 years $(\mathrm{IQR}=2-9)$. The vast majority of PWID reported using heroin (95.7\%) followed by cocaine (4.3\%), and no one reported using more than one type of a drug in a month preceding the survey (Table 1).

Injecting drugs at least once per day in a month before a survey was reported by $45.4 \%$ of PWID. Approximately two out of five respondents reported never sharing needles and syringes while injecting drugs. Sharing injecting equipment at least once during a month preceding the survey was reported by $14.1 \%$ of PWID while $8.4 \%$ shared injecting equipment last time they injected.

All but one respondent reported that they could obtain sterile injecting equipment when they needed it, either free-of-charge from harm reduction services or by buying them at pharmacies.

As sources of free-of-charge needles and syringes in the past 12 months respondents mentioned mobile outreach teams (8.0\%), primary health-care centers (17.9\%), and drop-in centers (53.5\%).

A substantial proportion of respondents $(60.8 \%)$ reported that they bought needles and syringes at pharmacies in the past 12 months.

During the past 12 months, $75.8 \%$ of PWID received risk-reduction counseling or some form of education about HIV prevention.

Table 2 shows the prevalence of needle and syringe sharing in the past month by socio-demographic and behavioral variables and results of bivariate and multivariate logistic regression.

After adjustment in the multivariate analysis, significantly higher odds of needle and syringe sharing were found among PWID who were older than 26 years $(\mathrm{aOR}=4.2$, 
Table 1 Sexual and injecting drug use behaviors in people who inject drugs in Podgorica, Montenegro, 2013

\begin{tabular}{|c|c|c|c|}
\hline & $n / N$ & Sample prevalence $\%^{\mathrm{a}}$ & $\begin{array}{l}\mathrm{RDS}^{\mathrm{b}} \text { prevalence } \\
\text { estimates } \%(95 \% \mathrm{CI})^{\mathrm{c}}\end{array}$ \\
\hline Currently has regular sexual partner & $217 / 401$ & 54.1 & $50.9(44.1-57.6)$ \\
\hline Used condom during last sex with regular partner ${ }^{d}$ & $100 / 396$ & 25.3 & $24.2(19.1-29.2)$ \\
\hline Discussed HIV status before having first sexual intercourse with regular partner ${ }^{d}$ & $127 / 401$ & 31.7 & $28.6(22.8-34.3)$ \\
\hline Had non-regular sexual partner in the past 12 months & $251 / 399$ & 62.9 & $62.2(56.2-68.4)$ \\
\hline $\begin{array}{l}\text { Used condoms consistently during sexual intercourse with non-regular partners } \\
\text { in the past } 12 \text { months }\end{array}$ & 109/251 & 43.4 & $46.4(37.1-55.8)$ \\
\hline \multicolumn{4}{|l|}{ Total number of sex partners in the past 12 months } \\
\hline $0-1$ & $123 / 392$ & 31.4 & $31.1(25.3-36.8)$ \\
\hline $2-3$ & $121 / 392$ & 30.9 & $34.0(27.2-40.8)$ \\
\hline $4-5$ & $63 / 392$ & 16.1 & $16.7(11.8-21.5)$ \\
\hline $6-10$ & $63 / 392$ & 16.1 & $13.7(9.6-17.8)$ \\
\hline$\geq 11$ & $22 / 392$ & 5.6 & $4.5(2.3-6.8)$ \\
\hline Ever had sexual intercourse for which received or gave money or drugs & $216 / 400$ & 54.0 & $48.8(42.0-55.6)$ \\
\hline Used condom during last sexual intercourse for which gave or received money or drugs & $169 / 216$ & 78.2 & $79.5(71.6-87.5)$ \\
\hline \multicolumn{4}{|l|}{ Used drugs before sexual intercourse in the past 12 months } \\
\hline Never & $16 / 398$ & 4.0 & $3.5(1.4-5.5)$ \\
\hline Rarely & $30 / 398$ & 7.5 & $11.9(7.4-16.4)$ \\
\hline Sometimes & $83 / 398$ & 20.9 & $22.4(17.0-27.8)$ \\
\hline Often & 269/398 & 62.2 & $62.2(55.9-68.4)$ \\
\hline \multicolumn{4}{|l|}{ Type of drugs used in the past month } \\
\hline Heroin & $381 / 401$ & 95.0 & $95.7(93.2-98.3)$ \\
\hline Cocaine & $20 / 401$ & 5.0 & $4.3(1.7-6.8)$ \\
\hline \multicolumn{4}{|l|}{ Frequency of injecting drugs in the past month } \\
\hline Once per month & $9 / 402$ & 2.2 & $3.0(0.6-5.4)$ \\
\hline Few times per month & $68 / 402$ & 16.9 & $23.1(15.7-30.4)$ \\
\hline Once per week & $9 / 402$ & 2.2 & $1.5(0.4-2.6)$ \\
\hline Several times per week & $91 / 402$ & 22.6 & $27.0(20.7-33.4)$ \\
\hline Once per day & $27 / 402$ & 6.7 & $5.1(2.8-7.3)$ \\
\hline Several times per day & $198 / 402$ & 49.3 & $40.3(33.3-47.3)$ \\
\hline Ever shared needles or syringes with someone else during drug injection & $260 / 402$ & 64.7 & $60.4(53.9-66.8)$ \\
\hline Shared needles or syringes with someone else in the past month & $57 / 399$ & 14.3 & $14.1(9.7-18.4)$ \\
\hline Shared needles or syringes with someone else in the last episode of injection & $31 / 401$ & 7.7 & $8.4(4.9-12.0)$ \\
\hline \multicolumn{4}{|l|}{ Received drug dependence treatment $\mathrm{e}^{\mathrm{e}}$} \\
\hline Currently & $25 / 399$ & 6.3 & $6.1(3.3-8.9)$ \\
\hline Not currently, but in the past & $160 / 399$ & 39.8 & $37.1(31.3-42.9)$ \\
\hline Never & 214/399 & 53.4 & $56.7(50.6-62.8)$ \\
\hline
\end{tabular}

anweighted estimates.

b Weighted estimates; RDS = respondent-driven sampling.

${ }^{\mathrm{c}} 95 \% \mathrm{Cl}=95 \%$ confidence interval.

${ }^{\mathrm{d}}$ Refers to current or most recent regular partner.

e Drug dependence treatment included opioid substitution therapy (as maintenance treatment), inpatient detoxification, outpatient drug dependence treatment, peer-based support groups.

95\% CI 1.4-14.8), females, though only marginally higher $(\mathrm{aOR}=2.5,95 \% \mathrm{CI} 1.0-6.1)$, those who injected drugs more frequently than once per day $(\mathrm{aOR}=2.4,95 \% \mathrm{CI}$
1.3-4.7), those who reported injecting drugs in parks or on streets in the past month $(\mathrm{aOR}=2.6,95 \%$ CI 1.3-5.4), those who reported not being provided with free-of- 
Table 2 Socio-demographic and behavioral correlates of sharing needles and syringes in the past month before a survey in Podgorica, Montenegro, 2013 (unweighted analysis)

\begin{tabular}{|c|c|c|c|c|}
\hline Variable & $n / N$ & $\begin{array}{l}\text { Prevalence of sharing needles } \\
\text { and syringes in the past month } \\
\text { before a survey (\%) with } 95 \% \mathrm{Cl}\end{array}$ & $\begin{array}{l}\text { Unadjusted OR } \\
(95 \% \mathrm{Cl})^{\mathrm{a}}\end{array}$ & $\begin{array}{l}\text { Adjusted OR } \\
(95 \% \mathrm{Cl})\end{array}$ \\
\hline Age group (years) & & & $p=0.054$ & $p=0.010^{b}$ \\
\hline$\leq 25$ & $4 / 64$ & $6.2(2.4-15.0)$ & 1.00 & 1.00 \\
\hline$\geq 26$ & $53 / 335$ & $15.8(12.3-20.1)$ & $2.81(0.98-8.08)$ & $4.16(1.44-14.77)$ \\
\hline Sex & & & $p=0.123$ & $p=0.039$ \\
\hline Male & $48 / 359$ & $13.4(10.2-17.3)$ & 1.00 & 1.00 \\
\hline Female & $9 / 40$ & $22.5(12.3-37.5)$ & $1.88(0.84-4.20)$ & $2.53(1.04-6.12)$ \\
\hline Monthly income & & & $p=0.041$ & $p=0.051$ \\
\hline$>300$ EUR & $4 / 68$ & $5.9(2.3-14.2)$ & 1.00 & 1.00 \\
\hline$\leq 301$ EUR & $52 / 329$ & $15.8(12.3-20.1)$ & $3.00(1.05-8.60)$ & $4.13(0.99-18.77)$ \\
\hline Frequency of injecting in the past month & & & $p=0.006$ & $p=0.008$ \\
\hline Once per day or less & $19 / 202$ & $9.4(6.1-14.2)$ & 1.00 & 1.00 \\
\hline More than once per day & $38 / 197$ & $19.3(14.4-25.4)$ & $2.30(1.27-4.15)$ & $2.44(1.25-4.73)$ \\
\hline Number of sex partners in the past 12 months & & & $p=0.023$ & $p=0.009$ \\
\hline$\leq 3$ & $26 / 242$ & $10.7(7.4-15.3)$ & 1.00 & 1.00 \\
\hline$\geq 4$ & $28 / 147$ & $19.1(13.5-26.1)$ & $1.95(1.09-3.48)$ & $2.35(1.24-4.46)$ \\
\hline Injected drugs in parks or on streets in the past month & & & $p=0.014$ & $p=0.010$ \\
\hline No & $41 / 330$ & $12.4(9.3-16.4)$ & 1.00 & 1.00 \\
\hline Yes & $16 / 66$ & $24.2(15.5-35.8)$ & $2.25(1.17-4.32)$ & $2.61(1.25-5.44)$ \\
\hline $\begin{array}{l}\text { Provided with free sterile needles and syringes in the } \\
\text { past } 12 \text { months }{ }^{c}\end{array}$ & & & $p=0.009$ & $p=0.008$ \\
\hline Yes & $49 / 381$ & $12.9(9.9-16.6)$ & 1.00 & 1.00 \\
\hline No & $6 / 16$ & $37.5(18.5-61.4)$ & $4.06(1.41-11.68)$ & $5.74(1.58-20.9)$ \\
\hline
\end{tabular}

${ }^{\mathrm{a}} \mathrm{OR}=$ odds ratio; $95 \% \mathrm{Cl}=95 \%$ confidence interval.

${ }^{b} p$ values represent significance test for heterogeneity across the variable.

${ }^{\mathrm{C}}$ This refers to being given free-of-charge needles and syringes by NGOs and/or the Primary Health Care Centre.

charge syringes and needles ( $\mathrm{aOR}=5.7,95 \%$ CI 1.6-20.9), and those who had four and more sexual partners in the last 12 months ( $\mathrm{aOR}=2.4,95 \% \mathrm{CI} 1.2-4.5)$.

\section{Discussion}

The HIV prevalence of 1.1\% found in Podgorica in 2013 is higher than in the surveys done in 2008 and 2011, though this finding still implies a low-level HIV epidemic in PWID. The prevalence of HCV was at a similar level in 2013 (53.0\%) as in 2008 and 2011.

Comparable HIV prevalence estimates were found in most recent surveys conducted in PWID in other countries of ex-Yugoslavia: 3\% in Belgrade, Serbia in 2005; $0 \%-0.5 \%$ in several cities in Bosnia and Herzegovina in 2011; 0 in Croatia in 2007 and in FYR Macedonia in 2010 [12-15]. However, epidemics of HCV in PWID are well established in these countries-the prevalence in the abovementioned surveys was $66 \%$ in Belgrade, Serbia, $23 \%-49 \%$ in several cities in Bosnia and Herzegovina, $44 \%-65 \%$ in several cities in Croatia, and 70\% in Skopje, FYR Macedonia [12-15].
It is encouraging that almost all respondents in this survey as in the 2008 and 2011 surveys $(96.8 \%$ and $99.6 \%$, respectively) reported that they could obtain sterile needles and syringes for injecting.

Sharing needles and syringes in a month prior to the survey was reported in 2013 by $14.1 \%$ of PWID, which is very similar to the findings from the survey carried out in 2011 (13.6\%) and substantially less than in 2008 (24.2\%), which might explain a stable prevalence of $\mathrm{HCV}$ in this time period.

However, there are several findings that show current gaps in HIV and HCV prevention and that have importance for further development of interventions for prevention of these infections in PWID in Montenegro.

Although a majority of PWID know where they can be tested for HIV, the HIV testing uptake is low-less than one in five respondents reported being tested for HIV in a year before the survey. A high proportion of respondents reported never being on drug dependence treatment. Interventions among PWID need to focus on ensuring better access to drug dependence treatment as it is highly 
effective in reducing injecting behaviors that put opioiddependent injectors at risk for HIV and blood-borne infections $[16,17]$.

Results of multivariate analysis highlighted that certain groups may benefit from stronger harm reduction programs that emphasize importance of consistent use of sterile injecting equipment, such as females who inject drugs (FWID), those who report higher number of sexual partners, and those who inject in outdoor settings.

It is well known that FWID are more difficult to reach with HIV prevention services compared to males and tend to engage in higher-risk injecting practices [18-21]. FWID usually depend on male partners for drugs and injections, leading to elevated drug and equipment sharing, and many engage in commercial sex. Given that FWID may be at higher risk of HIV, particularly via sexual transmission, there is a need to further explore barriers that they face in accessing harm reduction services. Separate prevalence assessment surveys can be done in FWID only to determine the level of HIV, HCV, and STIs in this sub-population and factors that expose them to higher risk of acquiring these infections.

There is evidence that PWID who engage in high-risk sexual behaviors might be more likely to engage in risky injection practices [22-25]. In our survey, almost a half of respondents reported ever selling or buying sex or exchanging it for drugs, and somewhat more than a half reported non-regular partners in the past 12 months and rather low condom use with such partners. Only a minority of respondents reported discussing HIV status before having the first intercourse with regular partners.

Of note is that in our study, older PWID reported significantly higher odds of needle and syringe sharing than younger PWID, which is a finding that has relevance for further intervention planning. Factors that put older PWID at risk of harmful injecting practices should be explored in Montenegro since research on the influence of age on the patterns of drug use mainly demonstrated that younger PWID are more likely than older PWID to share injecting equipment and engage in high-risk injecting behaviors [26-28].

Based on the results of this analysis, we recommend that free and confidential HIV testing and counseling should be more readily available for PWID as well as drug dependence treatment and sexual heath interventions that include screening for STIs, condom distribution programs, and promotion of safer sex practices. Since those who inject in outdoor settings had higher odds of needle and syringe sharing and a low proportion of PWID reported obtaining free-of-charge injecting equipment from outreach services, it is important to map sites in Podgorica where PWID gather so that harm reduction programs can be more effectively strengthened through outreach and mobile services.
This study was not without limitation. Self-reported behaviors are subject to social desirability and recall bias. Refusals to participate are challenging to measure in RDS and could have also biased the findings. RDS may have resulted in under-recruitment of certain types of participants, e.g., PWID with smaller injecting networks and women [29]. Better recruitment of females who inject drugs could have been achieved by having more female seeds. In the future HIV bio-behavioral surveys, efforts should be made to achieve more effective recruitment of FWID.

\section{Conclusion}

The current evidence shows that the HIV epidemic in PWID in Montenegro is still at the low level, though the HIV prevalence might have increased in the period 2011-2013. The HCV epidemic is well established in this population as slightly more than a half of recruited PWID were found to be HCV infected. It is of paramount importance that targeted and effective interventions based on the combination of behavioral, biomedical, and structural approaches are kept sustainable and continue to be scaled up.

\section{Endnote}

${ }^{\mathrm{a} C}$ Comprehensive knowledge of HIV meant knowing that proper and consistent use of condoms during sexual intercourse and having just one partner who is HIV negative and has no other partners can reduce the risk of getting HIV, knowing that a healthy-looking person can have HIV, knowing that it is possible to decrease the risk of HIV transmission by not sharing needles and other injecting equipment and rejecting the two most common local misconceptions about HIV transmission and prevention.

\section{Competing interests}

The authors declare that they have no competing interests.

\section{Authors' contributions}

$\mathrm{DL}$ conceived and designed the study, conducted the statistical analysis, interpreted the data, and drafted the manuscript. SB participated in the design, statistical analysis, and interpretation, drafting the manuscript, and supervised the collection of the data and data entry. BM participated in the design and coordination of the study and interpretation of the data. NT supervised the data entry and participated in the statistical analysis. ZV conducted the laboratory tests and participated in the interpretation of data. $\mathrm{IL}$ participated in the design of the study and interpretation of the data. IB participated in the interpretation of the data, drafting, and critically revising the manuscript. All authors read and approved the final manuscript.

\section{Acknowledgements}

This study was supported by a grant from the Global Fund to Fight AIDS, Tuberculosis and Malaria, through the United Nations Development Programme's HIV/AIDS Office in Podgorica. The authors thank all the study participants for their willingness to provide data and biological specimens. We also express our gratitude to the members of the NGO Juventas and the $\mathrm{IPH}$ staff for their assistance. 


\section{Author details}

${ }^{1}$ Institute for Public Health, Podgorica, Montenegro. ${ }^{2}$ HIV/AIDS Office, United Nations Development Programme, Podgorica, Montenegro. ${ }^{3}$ World Health Organization Collaborating Centre for HIV Surveillance, School of Medicine, University of Zagreb, Zagreb, Croatia.

Received: 11 November 2014 Accepted: 4 February 2015

Published online: 28 February 2015

\section{References}

1. Mathers BM, Degenhardt L, Phillips B, Wiessing L, Hickman M, Strathdee SA, et al. Global epidemiology of injecting drug use and HIV among people who inject drugs: a systematic review. Lancet. 2007:2008(372):1733-45.

2. Des Jarlais DC, Arasteh K, McKnight C, Hagan H, Perlman DC, Semaan S. Associations between herpes simplex virus type 2 and HCV With HIV among injecting drug users in New York City: the current importance of sexual transmission of HIV. Am J Public Health. 2011;101:1277-83.

3. Statistical Yearbook 2011. Podgorica: Statistical Office of the Republic of Montenegro; 2012.

4. Annual Report on HIV/AIDS in Montenegro. Podgorica: Institute for Public Health; 2014.

5. HIV/AIDS Strategy for the Republic of Montenegro 2005-2009. Podgorica: Government of the Republic of Montenegro; 2005

6. Lausevic D, Mugosa B, Vratnica Z, Terzic N, Strahinja R, Labovic I. Survey on HIV Risk Behaviours and Prevalence of HIV, HBV and HCV Among Injecting Drug Users in Montenegro in 2008. Podgorica: Institute for Public Health; 2008.

7. Lausevic D, Mugosa B, Vratnica Z. Survey on HIV Risk Behaviours and HIV, HBV and HCV Prevalence Among Injecting Drug Users in Montenegro in 2011. Podgorica: Institute for Public Health; 2011.

8. Heckathorn D. Respondent driven sampling: a new approach to the study of hidden populations. Soc Probl. 1997:44:174-99.

9. Magnani R, Sabin K, Saidel T, Heckathorn D. Review of sampling hard-to-reach and hidden populations for HIV surveillance. AIDS. 2005;19 suppl 2:S67-72.

10. Behavioural surveillance surveys: guidelines for repeated behavioural surveys in populations at risk of HIV. Arlington: Family Health International, 2000. Available at: http://www.who.int/hiv/strategic/en/bss_fhi2000.pdf Accessed on 29 January 2015.

11. Handcock MS, Fellows IE, Gile KJ. RDS Analyst: software for the analysis of respondent-driven sampling data, Version 0.42. Available at: http://hpmrg.org. Accessed on 22 September 2014.

12. Judd A, Rhodes T, Johnston LG, Platt L, Andjelkovic V, Simić D, et al. Improving survey method in sero-epidemiological studies of injecting drug users : a case example of two cross-sectional surveys in Serbia and Montenegro. BMC Infect Dis. 2009; 9-14. doi: 10.1186/1471-2334-9-14.

13. Bacak V, Dominkovic Z. Behavioral and Biological Surveillance Study Among Injection Drug Users in Bosnia and Herzegovina: A Respondent-Driven Sampling Survey. Banja Luka: Public Health Institute; 2012.

14. Vilibic-Cavlek T, Maric J, Katicic L, Kolaric B. Hepatitis C virus antibody status, sociodemographic characteristics and risk behavior among injecting drug users in Croatia. Cent Eur J Public Health. 2011;19:26-9.

15. Mikik V, Kuzmanovska G, Memeti S. Report from the Bio-Behavioural Survey Among Injecting Drug Users and Assessment Of Population Size of Injecting Drug Users in Macedonia, 2010. Skopje: Institute for Public Health of the Republic of Macedonia; 2011.

16. Committee on the Prevention of HIV Infection among Injecting Drug Users in High-Risk Countries. Preventing HIV Infection Among Injecting Drug Users in High Risk Countries: An Assessment of the Evidence. Washington DC: Institute of Medicine of the US National Academies; 2006.

17. Gowing L, Farrell M, Bornemann R, Sullivan L, Ali R. Substitution treatment of injecting opioid users for prevention of HIV infection. Cochrane Database Syst Rev. 2008;2, CD004145.

18. HIV/AIDS prevention and care for female injecting drug users. Vienna: United Nations Office on Drugs and Crime; 2006.

19. Benotsch EG, Somlai AM, Pinkerton SD, Kelly JA, Ostrovski D, Gore-Felton C et al. Drug use and sexual risk behaviours among female Russian IDUs who exchange sex for money or drugs. Int J STD AIDS. 2004;15:343-7.

20. Cleland CM, Des Jarlais DC, Perlis TE, Stimson G, Poznyak V. HIV risk behaviors among female IDUs in developing and transitional countries. BMC Public Health. 2007;7:271.
21. Des Jarlais DC, Feelemyer JP, Modi SN, Arasteh $\mathrm{K}$, Hagan H. Are females who inject drugs at higher risk for HIV infection than males who inject drugs: an international systematic review of high seroprevalence areas. Drug Alcohol Depend. 2012:124:95-107.

22. Assari S, Yarmohmmadi Vasel M, Tavakoli M, Sehat M, Jafari F, Narenjiha $H$, et al. Inconsistent condom use among Iranian male injection drug users. Front Psychiatry 2014, 181. doi: 10.3389/fpsyt.2013.00181. eCollection 2013.

23. Schumacher CM, Go VF, le Nam V, Latkin CA, Bergenstrom A, Celentano DD, et al. Social injecting and other correlates of high-risk sexual activity among injecting drug users in northern Vietnam. Int J Drug Policy. 2009;20:352-6.

24. Chikovani I, Bozicevic I, Goguadze K, Rukhadze N, Gotsadze G. Unsafe injection and sexual risk behavior among injecting drug users in Georgia. J Urban Health. 2011:88:736-48.

25. Neaigus A, Reilly KH, Jenness SM, Hagan H, Wendel T, Gelpi-Acosta C. Dual HIV risk: receptive syringe sharing and unprotected sex among HIV-negative injection drug users in New York City. AIDS Behav. 2013;17:2501-9.

26. Fennema JS, Van Ameijden EJ, Van Den Hoek A, Coutinho RA. Young and recent-onset injecting drug users are at higher risk for HIV. Addiction. 1997:92:1457-65.

27. Miller CL, Strathdee SA, Li K, Kerr T, Wood E. A longitudinal investigation into excess risk for blood-borne infection among young injection drug users. Am J Drug Alcohol Abuse. 2007;33:527-36.

28. Degenhardt $L$, Kinner SA, Roxburgh A, Black E, Bruno R, Fetherston J, et al. Drug use and risk among regular injecting drug users in Australia: does age make a difference? Drug Alcohol Rev. 2008;27:357-60.

29. Paquete $D$, Bryant J, de Wit J. Respondent driven sampling and the recruitment of people with small injecting networks. AIDS Behav. 2012;16:890-9.

\section{Submit your next manuscript to BioMed Central and take full advantage of:}

- Convenient online submission

- Thorough peer review

- No space constraints or color figure charges

- Immediate publication on acceptance

- Inclusion in PubMed, CAS, Scopus and Google Scholar

- Research which is freely available for redistribution 